Article

\title{
Nonclassical Symmetry Solutions for Non-Autonomous Reaction-Diffusion Equations
}

\author{
Bronwyn H. Bradshaw-Hajek \\ School of Information Technology and Mathematical Sciences, University of South Australia, \\ Mawson Lakes, SA 5095, Australia; Bronwyn.Hajek@unisa.edu.au
}

Received: 24 December 2018 ; Accepted: 6 February 2019; Published: 12 February 2019

\begin{abstract}
The behaviour of many systems in chemistry, combustion and biology can be described using nonlinear reaction diffusion equations. Here, we use nonclassical symmetry techniques to analyse a class of nonlinear reaction diffusion equations, where both the diffusion coefficient and the coefficient of the reaction term are spatially dependent. We construct new exact group invariant solutions for several forms of the spatial dependence, and the relevance of some of the solutions to population dynamics modelling is discussed.
\end{abstract}

Keywords: nonclassical symmetries; Q-conditional symmetries; Lie symmetries; exact solutions; reaction-diffusion equations; spatially-dependent diffusion; population dynamics

\section{Introduction}

Nonlinear reaction-diffusion equations have many applications in chemistry [1], microwave heating [2] and in various areas of biology, including population genetics [3,4], nerve impulses [5], cellular tissue growth [6], and calcium waves [7,8]. In more general situations, where the problem involves some spatial heterogeneity, the relevant reaction-diffusion equations may be written with spatially-dependent diffusion and reaction terms. Generally, this class of equations takes the form:

$$
u_{t}=\left(D(x, u) u_{x}\right)_{x}+R(x, u)
$$

where $D(x, u)$ is a diffusion coefficient and $R(x, u)$ is often a nonlinear reaction term.

There are many situations where the favorability (or otherwise) of a habitat or region of interest impacts the dynamics of a situation, and in some cases, it is appropriate to include this spatial inhomogeneity in the model equation. Contrell and Costner [9] included a step function change in the growth rate to model a problem in population dynamics, while Benson et al. [10] included a step function in the diffusion coefficient in a model for chick limb development. Other forms of spatial heterogeneity in population dynamics were investigated by Shigesada et al. [11], and Pacala and Roughgarden [12] included spatial inhomogeneities in the carrying capacity in a competing species model. In non-biological problems, the effect of spatial heterogeneity has been studied with regard to optical tomography problems [13] and flow through porous media (see Zappou and Knight [14], where the spatially-dependent diffusion is considered quadratic).

A number of exact analytic solutions exist when $D(u)=D$ is constant and $R(x, u)=Q(u)$ depends on $u$ only. Dorodnitsyn [15,16] presented the complete classical Lie point symmetry classification of reaction-diffusion equations. Travelling wave solutions have been found by Kametaka [17], McKean [18] and Rinzel [19], while periodic solutions have been found by Carpenter [20] and Hastings [21]. Other solutions presented in the context of population dynamics were given by Conte [22], Chen and $\mathrm{Gu}$ [23] and Kawahara and Tanaka [24]. The complete nonclassical symmetry classification was given by Arrigo et al. [25] and Clarkson and Mansfield [26], where it 
was shown that nonclassical symmetries can only be found when $Q(u)$ is cubic. Similar solutions can be found using Painlevé techniques [27]. Construction of Lie and nonclassical symmetries for autonomous reaction-diffusion equations with convective terms was initiated in [28] (see [29-31] for more results).

Analytic solutions also exist for a more general reaction term, $R(x, u)$, with constant diffusivity, $D(u)=D$. Joshi and Morrison [32] used Painlevé and Lie symmetry techniques to find solutions for specific forms of $R(x, u)$, while solutions to a similar class of equations were found by Hashemi and Nucci [33] using nonclassical symmetry analysis and the method of heir equations. Other exact solutions were presented by Bradshaw-Hajek et al. [4], Ivanova and Sophocleous [34] and Vaneeva and Zhalij [35] when $R(x, u)=r(x) Q(u)$.

In the case where the reaction term is a function of $u$ only, $R(x, u)=Q(u)$, while the diffusion coefficient is spatially dependent, $D(x, u)=D(x)$, analytic solutions have been found using nonclassical symmetry techniques [36,37].

Various symmetry techniques have been used to find solutions to more general autonomous reaction-diffusion equations with $D(x, u)=D(u)$ and $R(x, u)=R(u)$; see for example Arrigo and Hill [38], Goard and Broadbridge [39] and Cherniha et al. [31]. Travelling wave solutions have also been presented by Gilding and Kersner [40].

Fewer solutions exist for the case when both the diffusion and reaction terms are spatially dependent. Vaneeva et al. [41] used group analysis to construct exact solutions for equations of the form:

$$
f(x) u_{t}=\left(g(x) u_{x}\right)_{x}+h(x) u^{m} .
$$

In this paper, we use nonclassical symmetry methods to find some reductions and exact solutions for the case when $D(x, u)=D(x)$ is a spatially-dependent diffusion coefficient and $R(x, u)=r(x) Q(u)$ is a nonlinear reaction term with spatial dependence, i.e., equations of the type:

$$
u_{t}=\left(D(x) u_{x}\right)_{x}-r(x) Q(u)
$$

The nonclassical, or Q-conditional, symmetry technique is a conditional symmetry method that generalises Lie's classical point symmetry method. A classical point symmetry of a differential equation such as (1) is an invertible transformation of the dependent and independent variables of the form:

$$
\left.\begin{array}{rl}
t^{*} & =t+\epsilon T(t, x, u)+O\left(\epsilon^{2}\right) \\
x^{*} & =x+\epsilon X(t, x, u)+O\left(\epsilon^{2}\right) \\
u^{*} & =u+\epsilon U(t, x, u)+O\left(\epsilon^{2}\right)
\end{array}\right\}
$$

generated by the vector field:

$$
\Gamma=T(t, x, u) \frac{\partial}{\partial t}+X(t, x, u) \frac{\partial}{\partial x}+U(t, x, u) \frac{\partial}{\partial u}
$$

which leaves the governing equation invariant. For a second order partial differential equation such as Equation (1), this requirement can be written as:

$$
\left.\Gamma^{(2)}(F)\right|_{F=0}=0
$$

where $F\left(t, x, u, u_{t}, u_{x}, u_{x x}\right)$ is the governing Equation (1) and $\Gamma^{(2)}$ is the second prolongation of the base vector or symmetry generator, $\Gamma$ (see [42] for further detail). This requirement of invariance leads to an overdetermined, but linear system of PDEs for $T(t, x, u), X(t, x, u)$ and $U(t, x, u)$.

The nonclassical symmetry method was pioneered by Bluman and Cole [43]. This technique requires invariance of the governing equation as described above, augmented by the invariant surface condition:

$$
T(t, x, u) u_{t}+X(t, x, u) u_{x}=U(t, x, u)
$$


and its differential consequences:

$$
\begin{array}{r}
u_{t t} T+u_{x t} X=U_{[t]}, \\
u_{t x} T+u_{x x} X=U_{[x]},
\end{array}
$$

and so on. Here,

$$
U_{[t]}=D_{t}(U)-D_{t}(T) u_{t}-D_{t}(X) u_{x}
$$

$U_{[x]}$ is similarly defined, and $D_{t}$ and $D_{x}$ are total derivatives. The inclusion of this additional condition leads to an overdetermined system of nonlinear PDEs for $T(t, x, u), X(t, x, u)$ and $U(t, x, u)$ and can sometimes lead to the discovery of additional symmetries that cannot be found using the classical method. A strictly nonclassical symmetry solution of a differential equation may not be found using the classical point symmetries of the same equation.

Whilst in this paper, we consider infinitesimals that depend on the dependent and independent variables only, i.e., $(t, x, u)$, it would be interesting to consider a generalised vector field given by:

$$
\Gamma_{n}=T\left(t, x, u, u^{(1)}, \ldots, u^{(n)}\right) \frac{\partial}{\partial t}+X\left(t, x, u, u^{(1)}, \ldots, u^{(n)}\right) \frac{\partial}{\partial x}+U\left(t, x, u, u^{(1)}, \ldots, u^{(n)}\right) \frac{\partial}{\partial u}
$$

where $u^{(i)}$ represents all the $i$ th order partial derivatives of $u$ with respect to $t$ and $x$ (as suggested by an anonymous referee). The search for a generalised nonclassical symmetry would then require that the equation in question remain invariant (2) and that the invariant surface condition (3) and its differential consequences be satisfied. It is possible that this may extend the class of symmetry-invariant diffusion equations and broaden the forms of $D(x), r(x)$ and $Q(u)$ that allow new nonclassical symmetries and solutions to be found.

A symmetry found by either the classical or nonclassical method can be used to find the invariants of the equation, and subsequently, the number of independent variables in the original differential equation may be reduced; for equations such as (1), the reduced equation will be an ordinary differential equation. After solving the reduced equation and working back through the transformations, a solution to the original differential equation may be found.

Here, we search for forms of $D(x), r(x)$ and $Q(u)$ that allow new classical and nonclassical symmetries and solutions to be found. The paper is organised as follows: In Section 2, we list the nonclassical determining equations for Equation (1), find admissible forms of $D(x), r(x)$ and $Q(u)$ and give the infinitesimals that characterise some classical and nonclassical symmetry generators. The admissible forms of the arbitrary functions are given in their simplest forms, but may be generalised by simple scalings and translations of the dependent and independent variables where appropriate. In Section 3, we obtain a number of example solutions, while in Section 4, we make some final remarks and discuss the relevance to population dynamics of the solutions presented here.

\section{Nonclassical Symmetries}

For $1+1$ dimensional equations, the case where $T(t, x, u)=0$ is known to be unfeasible, as finding a nonclassical operator is equivalent to solving the original governing equation [44]. Therefore, without loss of generality, we set $T(t, x, u)=1$ and proceed to solve the determining equations for $X(t, x, u)$ and $U(t, x, u)$.

The action of the second prolongation of the base vector, $\Gamma$, on the differential Equation (1), together with the requirement of invariance (2), gives an expression relating $X(t, x, u), U(t, x, u), Q(u), D(x)$ and $r(x)$ and their derivatives. After making use of the invariant surface condition (3), we find that this expression may be separated into four determining 
equations according to the coefficients of powers of $u_{x}$. The nonclassical determining equations for Equation (1) are:

$$
\begin{aligned}
D r Q_{u} X-D_{x} r Q X+D r_{x} Q X-D r Q U_{u}+2 D r Q X_{x} & \\
-D^{2} U_{x x}+D U_{t}-D_{x} X U-D D_{x} U_{x}+2 D X_{x} U & =0 \\
-D D_{x x} X-D D_{x} X_{x}+2 D X_{u} U-2 D X X_{x}+D_{x} X^{2} & \\
+D_{x}^{2} X-D X_{t}-2 D^{2} U_{x u}+D^{2} X_{x x}+3 D r Q X_{u} & =0 \\
D^{2} U_{u u}+2 D D_{x} X_{u}+2 D X X_{u}-2 D^{2} X_{x u} & =0 \\
D^{2} X_{u u} & =0
\end{aligned}
$$

We have been unable to solve this set of determining equations completely; however, some solutions have been found. From Equation (7), we see that:

$$
X(t, x, u)=f_{1}(t, x) u+f_{2}(t, x) .
$$

Substituting this into Equation (6), we obtain a PDE that can be solved to give:

$$
\begin{aligned}
U(t, x, u)=- & \frac{1}{3 D(x)} f_{1}(t, x)^{2} u^{3}+\frac{\partial f_{1}(t, x)}{\partial x} u^{2}-\frac{D^{\prime}(x)}{D(x)} f_{1}(t, x) u^{2} \\
& -\frac{1}{D(x)} f_{1}(t, x) f_{2}(t, x) u^{2}+f_{3}(t, x) u+f 4(t, x) .
\end{aligned}
$$

After substitution into Equation (5), we find:

$$
\begin{aligned}
& \frac{2}{3} f_{1}^{3} u^{3}+\left(3 f_{1}^{2} D_{x}-4 f_{1} f_{1 x} D+2 f_{1}^{2} f_{2}\right) u^{2} \\
& +\left(3 f_{1 x x} D^{2}-3 f_{1} D D_{x x}-2 f_{1} D f_{2 x}-2 f_{1} f_{3} D\right. \\
& \left.\quad+3 f_{1} D_{x}^{2}-3 f_{1 x} D D_{x}+2 f_{1} f_{2} D_{x}-2 f_{1 x} f_{2} D+f_{1 t} D\right) u \\
& \quad+f_{2} D D_{x x}+f_{2 x} D D_{x}+2 f_{2} f_{2 x} D-2 f_{1} f_{4} D+2 f_{3 x} D^{2} \\
& \quad-f_{2 x x} D^{2}-f_{2} D_{x}^{2}-f_{2}^{2} D_{x}+f_{2 t} D-3 f_{1} r D Q=0 .
\end{aligned}
$$

Since all unknown functions apart from $Q(u)$ are functions of $x$ and /or $t$ and we wish to find the forms of $Q(u)$ that satisfy Equation (8), we see that either $Q(u)$ must be cubic or $f_{1}(t, x)=0$. These two cases are investigated below.

This outcome is identical to the case when both $D(x)=D$ and $r(x)=r$ are constants; the solutions in this constant case were presented by Arrigo et al. [25], and their application to population dynamics was discussed elsewhere [45,46].

\section{1. $Q(u) C u b i c$}

After setting $Q(u)=a u^{3}+b u^{2}+c u+d$ and $f_{1}(t, x) \neq 0$, Equation (8) can be separated in terms of powers of $u$, enabling the the functions $f_{i}(t, x)$ to be determined in terms of the arbitrary functions $D(x)$ and $r(x)$. Substituting these into Equation (4), we obtain two PDEs for $D(x)$ and $r(x)$. Setting $D(x)=D$ and $r(x)=r$ constant, we can obtain the result obtained by Arrigo et al. [25]. Setting $D(x)=D$ and leaving $r(x)$ arbitrary, we obtain the result presented in [4], while setting $r(x)=r$ and leaving $D(x)$ arbitrary, we obtain the results presented in [36,37].

If $D(x)$ and $r(x)$ are both arbitrary, we are able to find a solution to Equation (4); however, we have not been able to solve it in general. If $b=c=d=0$, i.e., $Q(u)=a u^{3}$, and the spatially-dependent diffusion and reaction terms are given by:

$$
D(x)=D_{0} x^{2} \quad \text { and } \quad r(x)=x^{2 n},
$$


we find a nonclassical generator characterised by the infinitesimals:

$$
\begin{gathered}
T=1, \quad X=\left[3 \sqrt{\frac{a D_{0}}{2}} x^{n} u+D_{0}(2 n-1)\right] x, \\
U=-\frac{1}{2}\left[3 a x^{2 n} u^{2}+3 \sqrt{2 a D_{0}} n x^{n} u+D_{0} n(n+1)\right] u .
\end{gathered}
$$

This nonclassical symmetry was first found by Louw and Moitsheki [47], where they considered the cases $n=1$ and $n \neq 1$. The resulting solutions were discussed in the context of population dynamics.

2.2. $f_{1}(t, x)=0$

Setting $f_{1}(t, x)=0$, Equations (4) and (5) become:

$$
\begin{gathered}
-\left(f_{3 x x} u+f_{4 x x}\right) D^{2}-\left(f_{3 x} u+f_{4 x}\right) D D_{x}+\left(f_{3 t} u+f_{4 t}\right) D \\
+\left(f_{3} u+f_{4}\right)\left(D r Q_{u}-f_{2} D_{x}+2 f_{2 x} D\right)-f_{2} D_{x} r Q \\
+f_{2} D r_{x} Q-f_{3} D r Q+2 f_{2 x} \operatorname{Dr} Q=0 \\
-f_{2} D D_{x x}-f_{2 x} D D_{x}-2 f_{2} f_{2 x} D+f_{2} D_{x}^{2}+f_{2}^{2} D_{x} \\
-2 f_{3 x} D^{2}+f_{2 x x} D^{2}-f_{2 t} D=0 .
\end{gathered}
$$

In order to find the admissible forms of the arbitrary functions $D(x), r(x)$ and $Q(u)$, we must consider the cases when $f_{3}(t, x)$ and $f_{4}(t, x)$ are either zero or nonzero.

Setting both functions to zero, $f_{3}(t, x)=f_{4}(t, x)=0$, we see that $Q(u)$ is a factor of all remaining terms in Equation (10) and does not appear at all in Equation (11),

$$
\begin{array}{r}
-f_{2} D_{x} r Q+f_{2} D r_{x} Q-f_{3} D r Q+2 f_{2 x} D r Q=0, \\
-f_{2} D D_{x x}-f_{2 x} D D_{x}-2 f_{2} f_{2 x} D+f_{2} D_{x}^{2}+f_{2}^{2} D_{x}+f_{2 x x} D^{2}-f_{2 t} D=0 .
\end{array}
$$

Since we are not interested in the case when $Q(u)=0$, any set of functions $f_{2}(t, x), D(x), r(x)$ that satisfies Equations (10) and (11) and forms a nonclassical symmetry will be valid for any form of the nonlinear reaction term $Q(u)$. In this case, $f_{2}(t, x)$ may be written in terms of $D(x)$ and $r(x)$ by solving Equation (12),

$$
f_{2}(t, x)=s(t) \sqrt{\frac{D(x)}{r(x)}} \quad \text { or } \quad f_{2}(t, x)=s(t) \sqrt{-\frac{D(x)}{r(x)}} .
$$

By substituting this into Equation (13), we find:

$$
2 D_{x x} D r^{2}+2 D^{2} r r_{x x}-3 D^{2} r_{x}^{2}-D_{x}^{2} r^{2}-4 s D r r_{x} \sqrt{ \pm \frac{D}{r}}+4 \frac{s_{t}}{s} D r^{2}=0
$$

Since $s(t)$ is the only time-dependent function, one possible way of finding solutions is to require that that $s(t)$ takes one of three different forms described below.

2.2.1. $f_{1}(t, x)=0$ and $s(t)=c_{1}$

In this case, $s(t)=c_{1} \neq 0$ is a constant, so that $f_{2}(t, x)$ is a function of $x$ only. In this case and using the second form of $f_{2}(t, x)$ from (14), a simple relationship between $D(x)$ and $r(x)$ cannot be found, but instead, we obtain a single ODE that must be satisfied by $D(x)$ and $r(x)$,

$$
2\left(D^{\prime \prime} r+r^{\prime \prime} D\right) r D-D^{\prime 2} r^{2}-3 r^{\prime 2} D^{2}-4 c_{1} D \sqrt{-D r} r^{\prime}=0 .
$$


This equation is satisfied by the combination:

$$
D(x)=-\left(c_{1} x-c_{2}\right) \ln x, \quad \text { and } \quad r(x)=\frac{c_{1} x-c_{2}}{\ln x}
$$

when $x>1$, while the infinitesimals characterising the corresponding symmetry generator are:

$$
T=1, \quad X=c_{1} \ln x, \quad U=0 .
$$

This is a strictly nonclassical symmetry since the classical determining equations are not satisfied unless the invariant surface condition (3) and its differential consequences are invoked. In addition, it is valid for any form of the nonlinear reaction term, $Q(u)$.

2.2.2. $f_{1}(t, x)=0$ and $s(t)=\frac{1}{c_{1} t+c_{2}}$

In this case, we find that $D(x)$ is related to $r(x)$ via:

$$
D(x)=\frac{c_{1}^{2} r(x)^{3}}{r^{\prime}(x)^{2}}
$$

and subsequently, we find from (15) that $r(x)$ must satisfy:

$$
r^{\prime \prime \prime} r^{\prime} r+\left(r^{\prime 2}-2 r r^{\prime \prime}\right) r^{\prime \prime}=0 .
$$

The form of $r(x)$ that satisfies this and the corresponding spatially-dependent diffusion are given by:

$$
r(x)=\gamma x^{\alpha} \quad \text { and } \quad D(x)=D_{0} \gamma x^{\alpha+2}
$$

where $D_{0}=c_{1}^{2} / \alpha^{2}$. The infinitesimals that characterise the symmetry generator are:

$$
T=1, \quad X=\frac{x}{\alpha t+c_{2}}, \quad U=0 .
$$

This symmetry is a generalised version of the Boltzmann scaling symmetry,

$$
\Gamma=x \frac{\partial}{\partial x}+2 t \frac{\partial}{\partial t}
$$

and satisfies the classical determining Equation (2); it is not a nonclassical symmetry.

2.2.3. $f_{1}(t, x)=0$ and $s(t)=\frac{1}{c_{1} \mathrm{e}^{t / c_{2}^{2}}+c_{2}}$

For this form of $s(t)$, we find that $D(x)$ is related to $r(x)$ via:

$$
D(x)=\frac{r(x)^{3}}{c_{2}^{2} r^{\prime 2}} .
$$

By substituting this relationship and the form of $s(t)$ into the remaining determining Equation (15), we find that $r(x)$ must satisfy:

$$
r^{\prime \prime \prime} r^{\prime} r^{3}+\left(r^{\prime 2} r^{2}-2 r^{\prime \prime} r^{3}\right) r^{\prime \prime}+r^{4}=0 .
$$


While we have not solved this equation completely, a solution to this equation and the corresponding spatially-dependent diffusion is:

$$
r(x)=\frac{1}{\ln x}, \text { and } \quad D(x)=D_{0} x^{2} \ln x,
$$

while the infinitesimals that characterise the symmetry generator are:

$$
T=1, \quad X=\frac{D_{0} x \ln x}{\beta \mathrm{e}^{D_{0} t}-1}, \quad U=0
$$

This symmetry satisfies the classical determining Equation (2) and so is not a strictly nonclassical symmetry; however, it is valid for any form of the nonlinear reaction term, $Q(u)$.

Each of the three reduction operators described above arises from setting $f_{3}(t, x)=f_{4}(t, x)=0$. We have also investigated the cases where either one or both of $f_{3}(t, x)$ and $f_{4}(t, x)$ are nonzero; however, we have been unable to find a solution to the determining equations in these cases.

The classical and nonclassical symmetries presented in this section are summarised in Table 1.

Table 1. Admissible forms of the nonlinear reaction terms and the spatially-dependent diffusion and reaction coefficients when Equation (1) admit classical (c) and nonclassical (n) symmetries.

\begin{tabular}{|c|c|c|c|}
\hline & $D(x), r(x), Q(u)$ & Symmetry & Infinitesimals \\
\hline \multirow[t]{3}{*}{1} & $D(x)=D_{0} x^{2}$ & $\mathrm{n}$ & $T=1$ \\
\hline & $r(x)=x^{2 n}$ & & $X=\left[3 \sqrt{\frac{a D_{0}}{2}} x^{n} u+D_{0}(2 n-1)\right] x$ \\
\hline & $Q(u)=a u^{3}$ & & $U=-\frac{1}{2}\left[3 a x^{2 n} u^{2}+3 \sqrt{2 a D_{0}} n x^{n} u+D_{0} n(n+1)\right] u$ \\
\hline \multirow[t]{3}{*}{$2(a)$} & $D(x)=-\left(c_{1} x-c_{2}\right) \ln x$ & $\mathrm{n}$ & \\
\hline & $r(x)=\frac{c_{1} x-c_{2}}{\ln x}$ & & $X=c_{1} \ln x$ \\
\hline & $Q(u)$ arbitrary & & $U=0$ \\
\hline \multirow[t]{3}{*}{$2(b)$} & $D(x)=D_{0} \gamma x^{\alpha+2}$ & c & $T=1$ \\
\hline & $r(x)=\gamma x^{\alpha}$ & & $X=\frac{x}{\alpha t+c_{2}}$ \\
\hline & $Q(u)$ arbitrary & & $U=0$ \\
\hline \multirow[t]{2}{*}{$2(\mathrm{c})$} & $\begin{array}{l}D(x)=D_{0} x^{2} \ln x \\
r(x)=\frac{1}{\ln x}\end{array}$ & c & $\begin{array}{l}T=1 \\
X=\frac{D_{0} x \ln x}{\beta \mathrm{e}^{D_{0} t}-1}\end{array}$ \\
\hline & $Q(u)$ arbitrary & & $U=0$ \\
\hline
\end{tabular}
The infinitesimals are also given in each case.

\section{Reduced Equations and Some Example Solutions}

In this section, we find the reduced equations for the nonclassical and Lie point symmetries found above, and in some cases, we give some new exact solutions.

3.1. $Q(u)=a u^{3}, D(x)=D_{0} x^{2}, r(x)=x^{2 n}$

The first case, where $Q(u)$ is cubic and $D(x)$ and $r(x)$ are given by Equation (9), was examined by Louw and Moitsheki [47]. They found the reduced equations for $n=1$ and $n \neq 1$ and provided analytic solutions in both cases. This class of equations (with $Q(u)$ cubic) is of relevance in population 
dynamics and can be used to describe changes in the gene frequency in a diploid sexually-reproducing population [45]. Louw and Moitsheki discussed briefly the relevance of the solutions to these types of problems.

\section{2. $Q(u)$ Arbitrary}

3.2.1. $Q(u)$ Arbitrary, $D(x)=-\left(c_{1} x-c_{2}\right) \ln x, r(x)=\frac{c_{1} x-c_{2}}{\ln x}$

If the spatially-dependent diffusion and reaction terms are given by the combination (16), while the nonlinear reaction term remains arbitrary, the invariant surface condition (3) and the nonclassical generator characterised by (17) can be used to eliminate $u_{t}$ from the original PDE (1) to obtain:

$$
\ln x u_{x x}+\frac{1}{x} u_{x}+\frac{1}{\ln x} Q(u)=0 .
$$

After multiplying through by $\ln x u_{x}$, this equation may be integrated once to give:

$$
u_{x}=\frac{\sqrt{-2 \int^{u} Q\left(u^{\prime}\right) d u^{\prime}}}{|\ln x|}
$$

which is separable. After separating, integration with respect to $x$ yields an exponential integral, while integration with respect to $u$ depends on the form of $Q(u)$. Here, we give two examples that are relevant to modelling in population dynamics: the classic Fisher-type or logistic reaction term, $Q(u)=a u(u-1)$, which is often used to model cell populations or other asexually-reproducing species, and the Huxley-type reaction term, $Q(u)=a u^{2}(u-1)$, which is more appropriate for a sexually-reproducing species.

For $Q(u)=a u(u-1)$, integration with respect to $u$ gives:

$$
-\frac{2}{\sqrt{a}} \tanh ^{-1}\left(\frac{1}{\sqrt{3}} \sqrt{3-2 u}\right)+c(t)=\int^{x} \frac{d x^{\prime}}{\ln x^{\prime}}
$$

where $c(t)$ may be determined by substituting this into the original PDE. Upon doing this, we find that $c(t)$ must satisfy $c^{\prime}(t)=c_{1}$, so that:

$$
u(x, t)=\frac{3}{2}\left[1-\tanh ^{2}\left(\frac{\sqrt{a}}{2}\left(c_{1} t+c_{3}-\int^{x} \frac{d x^{\prime}}{\ln x^{\prime}}\right)\right)\right]
$$

is a solution to Equation (1) when $Q(u)$ is quadratic and the spatial dependence of the diffusion and reaction terms are given by (16).

For $Q(u)=a u^{2}(u-1)$, integration with respect to $u$ gives:

$$
-\sqrt{\frac{3}{2 a}} \sqrt{\frac{4-3 u}{u}}+c(t)=\int^{x} \frac{d x^{\prime}}{\ln x^{\prime}}
$$

where $c(t)$ may be determined by substituting this into the original PDE. After substitution, we find that $c(t)$ must satisfy $c^{\prime}(t)=c_{1}$, so that:

$$
u(x, t)=4\left[\frac{2 a}{3}\left(c_{1} t+c_{3}-\int^{x} \frac{d x^{\prime}}{\ln x^{\prime}}\right)^{2}+3\right]^{-1}
$$

is a solution to Equation (1) when $Q(u)$ is cubic and the spatial dependence of the diffusion and reaction terms are given by (16). Solutions (21) and (22) are new nonclassical symmetry solutions to Equation (1) with the diffusion and reaction terms as specified. 
3.2.2. $Q(u)$ Arbitrary, $D(x)=D_{0} \gamma x^{\alpha+2}, r(x)=\gamma x^{\alpha}$

For this combination of spatially-dependent diffusion and reaction coefficient, the symmetry operator found above is a classical point symmetry; however, we still present some example solutions here. When $Q(u)$ is arbitrary and $D(x)$ and $r(x)$ are given by Equation (18), Equation (1) may be reduced to:

$$
D_{0} \gamma \phi^{2} u_{\phi \phi}+\left(D_{0} \gamma(\alpha+2) \phi-\phi^{1-\alpha}\right) u_{\phi}-\gamma Q(u)=0
$$

where $\phi=x\left(\alpha t-c_{2}\right)^{1 / \alpha}$. After introducing the transformation $y=\ln \phi$, this may be simplified to:

$$
D_{0} \gamma u_{y y}+\left(D_{0} \gamma(\alpha+1)-\mathrm{e}^{-\alpha y}\right) u_{\phi}-\gamma Q(u)=0 .
$$

It is difficult to progress any further without giving a specific form for the nonlinear reaction term, and in fact, there are very few forms of $Q(u)$ for which analytic solutions may be found. One example that does admit analytic solutions is if $Q(u)$ is linear. In this case, solutions may be written in terms of Kummer functions, Bessel functions (if $\alpha=-1$ ) or Whittaker functions (if $\alpha=-2$ ) [48]. In the case when $\alpha=-2$, the diffusion coefficient is constant, and the symmetry generator reduces to:

$$
\Gamma=\frac{\partial}{\partial t}+\frac{x}{c_{2}+2 t} \frac{\partial}{\partial x}
$$

which is equivalent to the classical Boltzmann scaling symmetry.

3.2.3. $Q(u)$ Arbitrary, $D(x)=D_{0} x^{2} \ln x, r(x)=\frac{1}{\ln x}$

When the spatially-dependent diffusion and reaction terms are given by combination (19) while the nonlinear reaction term remains arbitrary, the classical generator characterised by (20) may be used to reduced the original PDE (1) to:

$$
D_{0} \phi^{2} u_{\phi \phi}+D_{0}(\beta \phi+1) \phi u_{\phi}-Q(u)=0,
$$

where $\phi=\ln x /\left(\beta-\mathrm{e}^{-D_{0} t}\right)$. This equation may be simplified by introducing the transformation $y=\ln \phi$ to give:

$$
D_{0} u_{y y}+D_{0} \beta \mathrm{e}^{y} u_{y}-Q(u)=0
$$

No further progression can be made for general $Q(u)$, and we have not been able to find any solutions if $\beta \neq 0$. However, if $\beta=0$, Equation (23) can be integrated twice after multiplying by $u_{y}$ to obtain:

$$
\sqrt{\frac{2}{D_{0}}} y=\int^{u} \frac{d \tilde{u}}{\sqrt{\int^{\tilde{u}} Q(\bar{u}) d \bar{u}}} .
$$

The right-hand side can be evaluated for some forms of $Q(u)$, and once again, we provide two examples using the same forms as those above.

For a quadratic Fisher-type reaction term $Q(u)=a u(u-1)$, integration yields:

$$
\sqrt{\frac{2}{D_{0}}} y=\frac{2 \sqrt{2}}{\sqrt{a}} \tan ^{-1}\left(\frac{1}{\sqrt{3}} \sqrt{2 u-3}\right)+\bar{c}
$$

where $\bar{c}$ is a constant, and the other constant of integration has been set to zero. After rearranging and working back through the transformations, we find that a solution to the original PDE (1) with spatial dependence as given by (19) is:

$$
u(x, t)=\frac{3}{2} \sec ^{2}\left[\frac{\sqrt{a}}{2 \sqrt{D_{0}}}\left(\ln (-\ln x)+D_{0} t+c\right)\right]
$$


for $0<x<1$ and where $c=-\sqrt{D_{0}} \bar{c}$.

For a cubic Huxley-type reaction term, $Q(u)=a u^{2}(u-1)$, integration yields:

$$
\sqrt{\frac{2}{D_{0}}} y=\sqrt{\frac{3(3 u-4)}{a u}}+\bar{c}
$$

where $\bar{c}$ is a constant, and the other constant of integration has been set to zero. After rearranging and working back through the transformations, a solution to the original PDE (1) with spatial dependence as given by Equation (19) is:

$$
u(x, t)=4\left[3-\frac{2 a}{3 D_{0}}\left(c+\ln (-\ln x)+D_{0} t\right)^{2}\right]^{-1}
$$

where $c=-\left(3 D_{0} / 2 a\right) \bar{c}$.

\section{Discussion and Final Remarks}

In this paper, we have investigated a class of nonlinear reaction diffusion equations with arbitrary spatial dependence in both the diffusion coefficient and the coefficient of the reaction term. Although we have not solved the determining equations completely, we have found some new forms of the spatial dependence that admit nonclassical or classical point symmetries. For these forms of spatial dependence, we have found the reduced equation, and for some examples, we have constructed new analytic solutions. In this paper, we have considered traditional nonclassical symmetries, that is we have looked for infinitesimals that depend on the independent and dependent variables only $(t, x, u)$, and not on the derivatives of $u$. It would be interesting to discover if the forms of $D(x), r(x)$ and $Q(u)$ that allow construction of an analytic solution may be expanded by the use of generalised nonclassical symmetries as described in the Introduction. We leave this for future work.

Unfortunately, none of the spatial dependencies that we have found here match the step function dependence of the real-world applications discussed in the Introduction. However, we now discuss the application of one of the solutions found here to a model of potential relevance to population dynamics. Nonlinear reaction diffusion equations of type (1) can be used to describe changes in the frequency of a new recessive gene. The quadratic Fisher-type logistic nonlinearity can be used to describe this situation for an asexually-reproducing population, whereas the cubic Huxley-type nonlinearity is more appropriate for a sexually-reproducing diploid population. In this case, the dependent variable, $u(x, t)$, might represent the density of one of the genotypes in the population. Figure $1 \mathrm{a}, \mathrm{b}$ shows the spatial dependence given by (16). For the parameter set chosen, individuals diffuse more readily at the left side of the range, while at the right-hand side of the range, the diffusion coefficient becomes slightly negative, indicating aggregation. The strength of the reaction term also varies throughout the range, being greater at the right-hand end. Figure 1c,d shows the solutions presented in Equations (21) and (22) for quadratic and cubic nonlinearities, respectively. Interestingly, the behaviour of the population density is similar in both cases: the profile is similar, and the frequency decreases with time. However, the decrease in the quadratic Fisher-type case is much faster than the decrease in the cubic Huxley-type case. The final time shown for the quadratic case is (nondimensional) $t=1.5$, whereas the final time shown for the cubic case is (nondimensional) $t=2.5$. At these different times, the population profiles are of a similar magnitude. This difference in the time taken for the population density to decrease again highlights the importance of choosing an appropriate model for the situation under consideration [49]. 
(a)

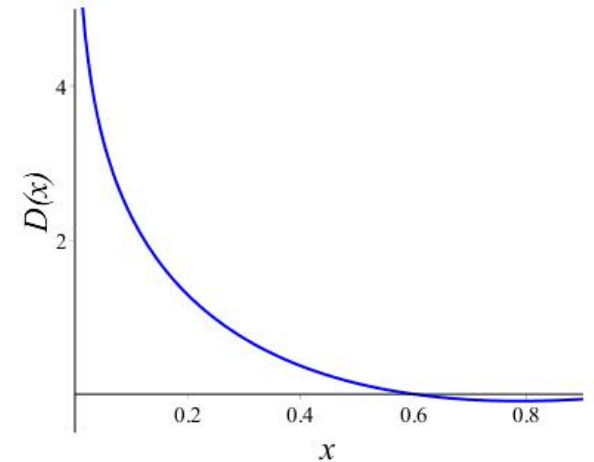

(c)

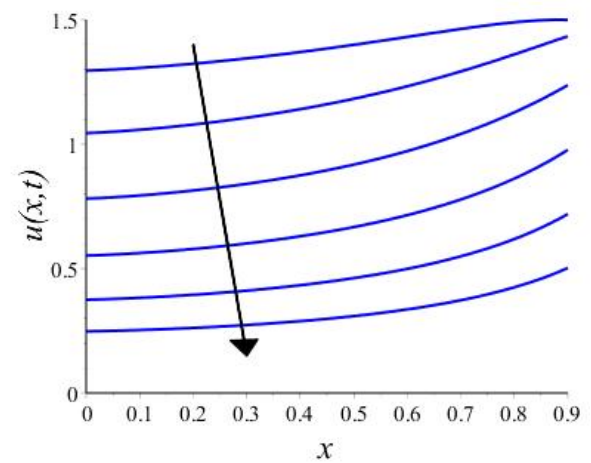

(b)

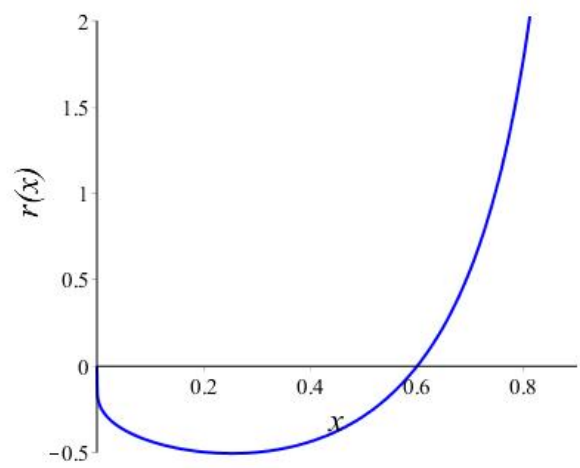

(d)

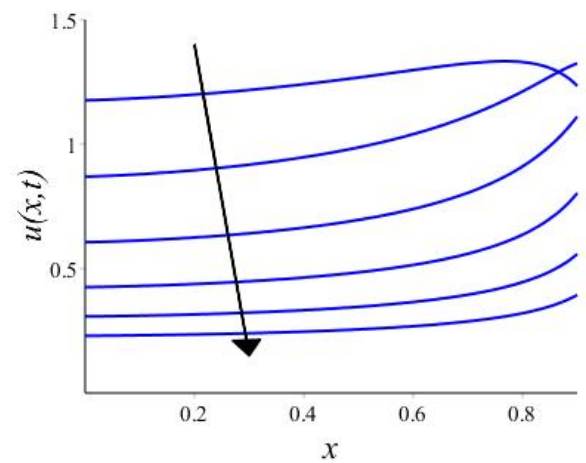

Figure 1. Plot of the spatial dependence and the example solutions arising from Symmetry 2(b). (a) Spatially-dependent diffusion coefficient. (b) Spatially-dependent coefficient of the reaction term. (c) Population density as given by the solution (21) for the quadratic Fisher-type reaction term $(t=0,0.3, \ldots, 1.5)$. (d) Population density as given by the solution (22) for the cubic Huxley-type reaction term $(t=0,0.5, \ldots, 2.5)$. In both cases, the arrow shows increasing time. In all plots, the parameter values are $a=0.6, c_{1}=-2, c_{2}=-1.2, c_{3}=-1$.

Funding: This research received no external funding.

Acknowledgments: The author would like to thank the anonymous referees for their useful comments on an earlier version of this paper.

Conflicts of Interest: The author declares no conflict of interest.

\section{References}

1. Aris, R. Mathematical Theory of Diffusion and Reaction in Permeable Catalysts I and II; Clarendon Press: Oxford, UK, 1975.

2. Hill, J.M.; Smyth, N.F. On the mathematical analysis of hot-spots arising from microwave heating. Math. Eng. Ind. 1990, 2, 267-278.

3. Fisher, R.A. The wave of advance of advantageous genes. Ann. Eugen. 1937, 7, 355-369. [CrossRef]

4. Bradshaw-Hajek, B.H.; Edwards, M.P.; Broadbridge, P.; Williams, G.H. Nonclassical symmetry solutions for reaction-diffusion equations with explicit spatial dependence. Nonlinear Anal. Theor. 2007, 67, 2541-2552. [CrossRef]

5. Scott, A.C. The electrophysics of a nerve fiber. Rev. Mod. Phys. 1975, 47, 487-533. [CrossRef]

6. Simpson, M.J.; Landman, K.A.; Hughes, B.D.; Newgreen, D.F. Looking inside an invasion wave of cells using continuum models: Proliferation is the key. J. Theor. Biol. 2006, 243, 343-360. [CrossRef] [PubMed]

7. Lane, D.C.; Murray, J.D.; Manoranjan, V.S. Analysis of wave phenomena in a morphogenetic mechanochemical model and an application to post-fertilization waves on eggs. Math. Med. Biol. 1987, 4, 309-331. [CrossRef] 
8. Bradshaw-Hajek, B.H.; Broadbridge, P. Analytic solutions for calcium ion fertilisation waves on the surface of eggs. Math. Med. Biol. 2019, accepted. [CrossRef] [PubMed]

9. Cantrell, R.S.; Cosner, C. The effects of spatial heterogeneity in population dynamics. J. Math. Biol. 1991, 29, 315-338. [CrossRef]

10. Benson, D.L.; Sherratt, J.A.; Maini, P.K. Diffusion driven instability in an inhomogeneous domain. Bull. Math. Biol. 1993, 55, 365-384. [CrossRef]

11. Shigesada, N.; Kawasaki, K.; Teramoto, E. Spatial segregation of interacting species. J. Theor. Biol. 1979, 79, 83-99. [CrossRef]

12. Pacala, S.W.; Roughgarden, J. Spatial heterogeneity and interspecific competition. Theor. Popul. Biol. 1982, 21, 92-113. [CrossRef]

13. Hielscher, A.H.; Klose, A.D.; Hanson, K.M. Gradient-based iterative image reconstruction scheme for time-resolved optical tomography. IEEE Trans. Med. Imaging 1999, 18, 262-270. [CrossRef] [PubMed]

14. Zoppou, C.; Knight, J.H. Analytical solution of a spatially variable coefficient advection-diffusion equation in up to three dimensions. Appl. Math. Model. 1999, 23, 667-685. [CrossRef]

15. Dorodnitsyn, V.A. Invariant solutions of the nonlinear heat equation with a source. Zhurnal Vychislitel'noi Matematiki i Matematicheskoi Fiziki 1982, 22, 1393-1400.

16. Galaktionov, V.A.; Dorodnitsyn, V.A.; Elenin, G.G.; Kurdyumov, S.P.; Samarskii, A.A. A quasilinear heat equation with a source: Peaking, localization, symmetry exact solutions, asymptotics, structures. J. Sov. Math. 1988, 41, 1222-1292. [CrossRef]

17. Kametaka, Y. On the nonlinear diffusion equation of Kolmogorov-Petrovskii-Piskunov type. Osaka J. Math. 1976, 13, 11-66.

18. McKean, H.P. Nagumo's equation. Adv. Math. 1970, 4, 209-223. [CrossRef]

19. Rinzel, J. Neutrally stable travelling wave solutions of nerve conduction equations. J. Math. Biol. 1975, 2, 205-217. [CrossRef]

20. Carpenter, G.A. Periodic solutions of nerve impulse equations. J. Math. Anal. Appl. 1977, 58, $152-173$. [CrossRef]

21. Hastings, S. The existence of periodic solutions to Nagumo's equations. Q. J. Math. 1974, 25, 369-378. [CrossRef]

22. Conte, R. Universal invariance properties of Painlevé analysis and Bäcklund transformation in nonlinear partial differential equations. Phys. Lett. A 1988, 134, 100-104. [CrossRef]

23. Chen, D.Y.; Gu, Y. Cole-Hopf quotient and exact solutions of the generalised Fitzhugh-Nagumo equations. Acta Math. Sci. 1999, 19, 7-14. [CrossRef]

24. Kawahara, T.; Tanaka, M. Interactions of travelling fronts: An exact solution of a nonlinear diffusion equation. Phys. Lett. A 1983, 97, 311-314. [CrossRef]

25. Arrigo, D.; Hill, J.M.; Broadbridge, P. Nonclassical symmetry reductions of the nonlinear diffusion equation with a nonlinear source. IMA J. Appl. Math. 1994, 52, 1-24. [CrossRef]

26. Clarkson, P.A.; Mansfield, E.L. Symmetry reductions and exact solutions of a class of nonlinear heat equations. Physica D 1994, 70, 250-288. [CrossRef]

27. Chen, Z.X.; Guo, B.Y. Analytic solutions of the Nagumo equation. IMA J. Appl. Math. 1992, 48, $107-115$.

28. Cherniha, R.; Serov, M. Symmetries, ansätze and exact solutions of nonlinear second-order evolution equations with convection terms. Eur. J. Appl. Math. 1998, 9, 527-542. [CrossRef]

29. Cherniha, R. New Q-conditional symmetries and exact solutions of some reaction-diffusion-convection equations arising in mathematical biology. J. Math. Anal. Appl. 2007, 326, 783-799. [CrossRef]

30. Cherniha, R.; Serov, M. Symmetries, ansätze and exact solutions of nonlinear second-order evolution equations with convection terms, II. Eur. J. Appl. Math. 2006, 17, 597-605. [CrossRef]

31. Cherniha, R.; Serov, M.; Pliukhin, O. Nonlinear Reaction-Diffusion-Convection Equations: Lie and Conditional Symmetry, Exact Solutions and Their Applications; CRC Press: Boca Raton, FL, USA, 2018.

32. Joshi, N.; Morrison, T. New exact solutions of spatially and temporally varying reaction-diffusion equations. Anal. Appl. 2008, 6, 371-381. [CrossRef]

33. Hashemi, M.S.; Nucci, M.C. Nonclassical symmetries for a class of reaction diffusion equations: the method of Heir-equations. J. Nonlinear Math. Phys. 2013, 20, 44-60. [CrossRef] 
34. Ivanova, N.M.; Sophocleous, C. On nonclassical symmetries of generalized Huxley equations. In Proceedings of 5th International Workshop "Group Analysis of Differential Equations and Integrable Systems" , Protaras, Cyprus, 6-10 June 2010; pp. 91-98.

35. Vaneeva, O.; Zhalij, A. Group classification of variable coefficient quasilinear reaction-diffusion equations. Publications de l Institut Mathematique-Beograd 2013, 94, 81-90. [CrossRef]

36. Moitsheki, R.J.; Bradshaw-Hajek, B.H. Symmetry analysis of a heat conduction model for heat transfer in a longitudinal rectangular fin of a heterogeneous material. Commun. Nonlinear Sci. 2013, 18, 2374-2387. [CrossRef]

37. Bradshaw-Hajek, B.H.; Moitsheki, R.J. Symmetry solutions for reaction-diffusion equations with spatially dependent diffusivity. Appl. Math. Comput. 2015, 254, 30-38. [CrossRef]

38. Arrigo, D.J.; Hill, J.M. Nonclassical symmetries for nonlinear diffusion and absorption. Stud. Appl. Math. 1995, 94, 21-39. [CrossRef]

39. Goard, J.M.; Broadbridge, P. Nonclassical symmetry analysis of nonlinear reaction-diffusion equations in two spatial dimensions. Nonlinear Anal. Theor. 1996, 26, 735-754. [CrossRef]

40. Gilding, B.H.; Kersner, R. Travelling Waves in Nonlinear Diffusion-Convection Reaction; Birkhäuser: Basel, Switzerland, 2004

41. Vaneeva, O.O.; Popovych, R.O.; Sophocleous, C. Enhanced group analysis and exact solutions of variable coefficient semilinear diffusion equations with a power source. Acta Appl. Math. 2009, 106, 1-46. [CrossRef]

42. Olver, P.J. Applications of Lie Group to Differential Equations; AMS No 107; Springer: New York, NY, USA, 1982.

43. Bluman, G.W.; Cole, J.D. The general similarity solution of the heat equation. J. Math. Mech. 1969, $18,1025-1042$.

44. Zhdanov, R.Z.; Lahno, V.I. Conditional symmetry of a porous medium equation. Physica D 1998, 122, 178-186. [CrossRef]

45. Broadbridge, P.; Bradshaw, B.H.; Fulford, G.R.; Aldis, G.K. Huxley and Fisher equations for gene propagation: An exact solution. ANZIAM J. 2002, 44, 11-20. [CrossRef]

46. Bradshaw-Hajek, B.H.; Broadbridge, P. A robust cubic reaction-diffusion system for gene propagation. Math. Comput. Model. 2004, 39, 1151-1163. [CrossRef]

47. Louw, K.; Moitsheki, R.J. Group-invariant solutions for the generalised Fisher type equation. Nat. Sci. 2015, 7, 613-624. [CrossRef]

48. Polyanin, A.D.; Zaitsev, V.F. Handbook of Exact Solutions for Ordinary Differential Equations; Chapman and Hall/CRC: Boca/Raton, FL, USA, 2003.

49. Broadbridge, P.; Bradshaw-Hajek, B.H. Exact solutions for logistic reaction-diffusion equations in biology. Z. Angew. Math. Phys. 2016, 67, 93. [CrossRef] 